\title{
Predator-Prey Model with Prey Harvesting, Holling Response Function of Type III and SIS Disease
}

\author{
Jean Jules Tewa*, Ramses Djidjou Demasse ${ }^{\dagger}$ and Samuel Bowong ${ }^{\ddagger}$ \\ * National Advanced School of Engineering, University of Yaounde I \\ , UMI 209 UMMISCO, GRIMCAPE, Yaounde, Cameroon \\ Email: tewajules@gmail.com \\ $\dagger$ Faculty of Science, University of Yaounde I \\ UMI 209 UMMISCO, GRIMCAPE, Yaounde, Cameroon \\ Email: dramsess@yahoo.fr \\ $\ddagger$ Faculty of Science, University of Douala \\ UMI 209 UMMISCO, GRIMCAPE, Yaounde, Cameroon \\ Email: sbowong@gmail.fr
}

Received: 28 June 2012, accepted: 23 October 2012, published: 28 December 2012

\begin{abstract}
The populations of prey and predator interact with prey harvesting. When there is no predator, the logistic equation models the behavior of the preys. For interactions between preys and predators, we use the generalized Holling response function of type III. This function which models the consumption of preys by predators is such that the predation rate of predators increases when the preys are few and decreases when they reach their satiety. Our main goal is to analyze the influence of a SIS infectious disease in the community. The epidemiological SIS model with simple mass incidence is chosen, where only susceptibles and infectious are counted. We assume firstly that the disease spreads only among the prey population and secondly that it spreads only among the predator population. There are many bifurcations as: Hopf bifurcation, transcritical bifurcation and saddle-node bifurcation. The results indicate that either the disease dies out or persists and then, at least one population can disappear because of infection. For some particular choices of the parameters however, there exists endemic equilibria in which both populations survive. Numerical simulations on MATLAB and SCILAB are used to illustrate our results.
\end{abstract}

Keywords-Predator; Prey; Infectious disease; Response function; Bifurcation; Global Stability

\section{INTRODUCTION}

There are many epidemiological or ecological models [6], [7], [8], [9], [10], [11], [5] in the literature and also many models which encompass the two fields [3], [4], [8], [9], [10], [11], [12]. Dynamic models for infectious diseases are mostly based on compartment structures that were initially proposed by Kermack and McKendrick $(1927,1932)$ and developed later by many other researchers.

The main questions regarding population dynamics concern the effects of infectious diseases in regulating natural populations, decreasing their population sizes, reducing their natural fluctuations, or causing destabilizations of equilibria into oscillations of the population states. With the Holling function response of type III, it is well known that the predators increase their searching activity when the prey density increases.

Generally, if $x$ denotes the density of prey population, the Holling function of type $\mathrm{I}$ is $\phi_{1}(x)=r x$ where $r$ is the intrinsic growth rate of preys. The Holling function of type II is $\phi_{2}(x)=\frac{B \omega_{0} x}{1+B \omega_{1} x}$, where $\omega_{0}$ and $\omega_{1}$ denote respectively the time taking by a predator to search and 
capture preys, $B$ is the predation rate per unit of time. In the models considered in this work, the Holling function of type III is used for interactions between predators and preys : $\phi_{3}(x)=\frac{m x^{2}}{a x^{2}+b x+1}$ [2], where $m$ and $a$ are positive constants, $b$ is an arbitrary constant. This function models the consumption of preys by predators. It is well known that with this function, the predation rate of predators increases when the preys are few and decreases when they reach their satiety (a predator increases his searching activity when the prey density increases). The functions $\phi_{1}, \phi_{2}$ and $\phi_{3}$ are respectively also referred to as Lotka-Volterra, Michaelis-Menten and sigmoidal response functions. Generally, there are more macroparasitic infections which can affect only preys, only predators or both preys and predators. Our goal in this paper is to analyze the influence of a SIS infectious disease which spreads only in one of the two populations. The models considered and analyzed here are different from all the models in the literature. Moreover, we use numerical simulations on MATLAB and SCILAB to illustrate our results.

\section{The Model Formulation}

The model (s1) is obtained from the classic LotkaVolterra model with simple mass action when the disease spreads only inside the prey population. In this model, the infected preys do not reproduce and there is no disease related mortality. The model (s2) is obtained when the disease spreads only inside the predator population. These models are respectively

$$
\begin{aligned}
& \left\{\begin{array}{l}
\dot{x}=\tilde{r}\left(1-\frac{x}{\tilde{k}}\right) x-\frac{\tilde{m} x^{2} y}{\tilde{a} x^{2}+\tilde{b} x+1}-\tilde{\lambda} x z \\
\quad+\tilde{\gamma} z-\tilde{h}_{1}, \\
\dot{z}=\tilde{\lambda} x z-\tilde{\gamma} z-\frac{\tilde{m} z^{2} y}{\tilde{a} z^{2}+\tilde{b} z+1}, \quad(s 1) \\
\dot{y}=\frac{\tilde{c} \tilde{m} x^{2} y}{\tilde{a} x^{2}+\tilde{b} x+1}-\frac{\tilde{m} z^{2} y}{\tilde{a} z^{2}+\tilde{b} z+1}-\tilde{d} y, \\
x \geq 0, z \geq 0, y \geq 0,
\end{array}\right. \\
& \left\{\begin{aligned}
\dot{x}= & \left(1-\frac{x}{\tilde{k}}\right) x-\frac{\tilde{m} x^{2} y}{\tilde{a} x^{2}+\tilde{b} x+1}-\frac{\tilde{\eta}_{1} x^{2} \omega}{\tilde{a} x^{2}+\tilde{b} x+1} \\
& -\tilde{h}_{1}, \\
\dot{y}= & \frac{\tilde{c} \tilde{m} x^{2} y}{\tilde{a} x^{2}+\tilde{b} x+1}-\tilde{d} y-\tilde{\delta} y \omega+\tilde{\mu} \omega, \\
\dot{\omega}= & \frac{\tilde{e} \tilde{m} x^{2} \omega}{\tilde{a} x^{2}+\tilde{b} x+1}+\tilde{\delta} y \omega-(\tilde{\mu}+\tilde{d}) \omega, \\
x \geq & 0, y \geq 0, \omega \geq 0 .
\end{aligned}\right.
\end{aligned}
$$

where the variables $z$ and $\omega$ denotes respectively the infected preys and infected predators, $\tilde{r}$ denotes the intrinsic growth rate of preys, $\tilde{d}$ is the natural death rate of predators, $\tilde{k}$ is the capacity of environment to support the growth of preys, $\tilde{h}_{1}$ is the rate of preys's harvesting, $\tilde{\gamma}$ and $\tilde{\mu}$ are the recover rates of infected preys and infected predators respectively, $\tilde{\lambda}$ is the adequate contact rate between susceptible preys and infected preys while $\tilde{\delta}$ is the adequate contact rate between susceptible predators and infected predators. We also assume that infected predators still can catch preys at a different rate $\tilde{\eta}_{1}$ than sound ones. The parameter $\tilde{\eta}_{1}$ can be thought to be less than $\tilde{m}$, if the disease affects the ability in hunting of the predators or larger than $\tilde{m}$, if we want to emphasize that the interactions with infected predators cause the preys to die for the disease even if they are not caught. $\tilde{a}$ and $\tilde{b}$ are positive constants. $\tilde{m}>0$ and $\tilde{m}_{1}>0$ denote the adequate predation rate between predators and preys. $\tilde{c}$ and $\tilde{e}$ denote the conversion coefficients. $\tilde{m}_{2}$ can be negative (conversion of prey's biomass into predator's biomass) or positive (bad effect of the infected preys for the predator population due to disease).

Trough the linear transformation and time scaling $(X, Z, Y, W, T)=\left(\frac{x}{\tilde{k}}, \frac{z}{\tilde{k}}, \frac{y}{\tilde{c} \tilde{k}}, \frac{\omega}{\tilde{e} \tilde{k}}, \tilde{c} \tilde{m} \tilde{k}^{2} t\right)$, the following simplified systems are obtained from (s1) and (s2),

$$
\left\{\begin{array}{l}
\dot{x}=\rho x(1-x)-p(x) y-\lambda x z+\gamma z-h_{1} \\
\dot{z}=\lambda x z-\gamma z-m_{1} p(z) y \\
\dot{y}=p(x) y-m_{2} p(z) y-d y \\
x \geq 0 ; y \geq 0 ; z \geq 0
\end{array}\right.
$$

$$
\left\{\begin{array}{l}
\dot{x}=\rho x(1-x)-p(x) y-\eta_{1} p(x) \omega-h_{1}, \\
\dot{y}=p(x) y-d y-\delta y \omega+\mu \omega \\
\dot{\omega}=e p(x) \omega+\delta_{1} y \omega-\mu_{1} \omega \\
x \geq 0 ; y \geq 0 ; \omega \geq 0
\end{array}\right.
$$

where the parameters are defined as follow

$$
\begin{aligned}
& \rho=\frac{\tilde{r}}{\tilde{c} \tilde{m} \tilde{k}^{2}}, \eta_{1}=\frac{\tilde{\eta}_{1} \tilde{e}}{\tilde{c} \tilde{m}}, \eta_{2}=\frac{\tilde{\eta}_{2} \tilde{e}}{\tilde{c} \tilde{m}}, h_{1}=\frac{\tilde{h_{1}}}{\tilde{c} \tilde{m} \tilde{k}^{3}}, \lambda=\frac{\tilde{\lambda}}{\tilde{c} \tilde{m} \tilde{k}}, \\
& \gamma=\frac{\tilde{\gamma}}{\tilde{c} \tilde{m} \tilde{k}^{2}}, m_{1}=\frac{\tilde{m}_{1}}{\tilde{m}}, m_{2}=\frac{\tilde{m}_{2}}{\tilde{c} \tilde{m}}, m_{3}=\frac{\tilde{m}_{3}}{\tilde{c} \tilde{m}}, d=\frac{d}{\tilde{c} \tilde{m} \tilde{k}^{2}}, \\
& \delta=\frac{\tilde{\delta} \tilde{e}}{\tilde{c} \tilde{m} \tilde{k}}, \mu=\frac{\tilde{\mu} \tilde{e}}{\tilde{c}^{2} \tilde{m} \tilde{k}^{2}}, e=\frac{\tilde{e}}{\tilde{c}}, \delta_{1}=\frac{\tilde{\delta}}{\tilde{m} \tilde{k}}, \mu_{1}=\frac{\tilde{\mu}+\tilde{d}}{\tilde{c} \tilde{m} \tilde{k}^{2}}, \\
& a=\tilde{a} \tilde{k}^{2}, b=\tilde{b} \tilde{k}, p(x)=\frac{x^{2}}{a x^{2}+b x+1} .
\end{aligned}
$$

Systems (1) and System (2) are new and different from all the models in the literature. These models without disease give us the same system which has been analyzed without disease in [1]. 


\section{RESULTS}

A. Results for the Model (1) with Disease only in Prey Population

Let us set $u_{1}(x)=\frac{\rho x(1-x)-h_{1}}{(1+m) p(x)-m d}, R_{0}=$ $\frac{m_{2}(p(\eta)-d) u_{1}^{2}(\eta)}{a(p(\eta)-d)^{2} u_{1}^{2}(\eta)+b(\lambda \eta-\gamma)(p(\eta)-d) u_{1}(\eta)+(\lambda \eta-\gamma)^{2}}$ the basic reproduction number, and

$$
\begin{aligned}
& x_{1}=\frac{1-\sqrt{1-4 \frac{h_{1}}{\rho}}}{2}, x_{2}=\frac{1+\sqrt{1-4 \frac{h_{1}}{\rho}}}{2}, \\
& x_{z}=\frac{\gamma}{\lambda}, x_{0}=\frac{1}{2}, z_{0} \in \mathbb{R}_{+}^{*},
\end{aligned}
$$

the expressions of the positive real values $x_{0}, x_{1}, x_{2}, x_{z}$.

Theorem 1: The equilibrium points of System (1), according to the values of the parameters, are given as follow :

- When $h_{1}>\frac{\rho}{4}$, then there is no equilibrium point.

- When $h_{1}=\frac{\rho}{4}$, then the unique equilibrium is $B_{0}\left(x_{0}, 0,0\right)$ which is a double point if $d \neq$ $\frac{1}{a+2 b+4}$ and triple point if $d=\frac{1}{a+2 b+4}$.

- When $h_{1}<\frac{\rho}{4}$ and $a d \geq 1$, then the equilibria are $B_{1}\left(x_{1}, 0,0\right)$ and $B_{2}\left(x_{2} ; 0 ; 0\right)$.

- When $h_{1}<\frac{\rho}{4}$; ad $<1$ and $x_{3}=x_{1}$, then $B_{1}\left(x_{1}, 0,0\right)$ is a double point and $B_{2}\left(x_{2}, 0,0\right)$ exists.

- When $h_{1}<\frac{\rho}{4}$; ad $<1$ and $x_{3}=x_{2}$, then $B_{1}\left(x_{1}, 0,0\right)$ is simple and $B_{2}\left(x_{2}, 0,0\right)$ is a double point.

- When $h_{1}<\frac{\rho}{4} ; a d<1$ and $\left.x_{3} \in\right] x_{1} ; x_{2}[$, then $B_{1}\left(x_{1}, 0,0\right) ; \stackrel{4}{B}_{2}\left(x_{2}, 0,0\right)$ and $B_{3}\left(x_{3}, 0, y_{3}\right)$ exist, where $y_{3}=\frac{\rho x_{3}\left(1-x_{3}\right)-h_{1}}{d}>0$.

- When $h_{1}<\frac{\rho}{4} ; a d<1$ and $x_{3} \in\left[0 ; x_{1}[\cup] x_{2} ;+\infty[\right.$, then $B_{1}\left(x_{1}, 0,0\right)$ and $B_{2}\left(x_{2}, 0,0\right)$ exist.

- When $h_{1}<\frac{\rho}{4} ;$ ad $\left.<1 ; x_{4} \in\right] \eta ; x_{2}\left[, x_{2}>\right.$ $\max \left(x_{3} ; \frac{\gamma}{\lambda}\right)$ and $R_{0}>1$, then $B_{1}\left(x_{1}, 0,0\right)$; $B_{2}\left(x_{2}, 0,0\right)$ and $B_{4}\left(x_{4}, z_{4}, y_{4}\right)$ exist, where $x_{4}>0$, $z_{4}>0$ and $y_{4}>0$.

Proof: These equilibria are obtained by setting the right hand side of (1) equals to zero. For $y=0$ one has equation $\rho x^{2}-\rho x+h_{1}=0$. Then we have $B_{0}, B_{1}$ and $B_{2}$. For $z=0$, one has $p(x)=d \Longleftrightarrow(1-a d) x^{2}-$ $b d x-d=0$. We deduce $x_{3}$ and then $B_{3}$. The condition for existence of $B_{4}$ is $p(z)=\frac{1}{m_{2}}(p(x)-d)>0$ ie $p(x)-d>0 \Longleftrightarrow a d<1$ and $x \in] x_{3},+\infty[$.

Concerning the stability of these equilibria, the following theorem hold.

Theorem 2: Let's consider System (1).

- The equilibria $B_{0}$ and $B_{1}$ are always unstable.

- The equilibrium $B_{2}$ is stable if one of the following conditions is satisfied : $h_{1}<\frac{\rho}{4}, \frac{\gamma}{\lambda} \geq x_{2}$ and $p\left(x_{2}\right) \leq d$, or $h_{1}<\frac{\rho}{4}, \frac{\gamma}{\lambda}<x_{2}, p\left(x_{2}\right)=d$ and $p^{\prime \prime}\left(x_{2}\right) \leq 0$.

- The equilibrium $B_{3}$ is stable if one of the following conditions is satisfied. $h_{1}<\frac{\rho}{4}$, ad $\left.<1, x_{3} \in\right] x_{1} ; x_{2}[$ and $x_{3}=\frac{\gamma}{\lambda}$, or $h_{1}<\frac{\rho}{4}$, ad $\left.<1, x_{3} \in\right] x_{1} ; x_{2}\left[, x_{3}<\right.$ $\frac{\gamma}{\lambda}$ and $d>\frac{1}{a+2 b+4}$, or $h_{1}<\frac{\rho}{4}, a d<1, x_{3} \in$ ]$x_{1} ; x_{2}\left[, x_{3}<\frac{\gamma}{\lambda}, d<\frac{1}{a+2 b+4}\right.$ and $\chi_{0}\left(x_{3}\right)<0$, where $\chi_{0}\left(x_{3}\right)$ is the eigenvalue of $x_{3}$.

- The equilibrium point $B_{4}\left(x_{4}, z_{4}, y_{4}\right)$ is asymptotically stable if and only if the following conditions hold : $a_{2}<0 ; a_{2} a_{1}+a_{0}>0$ and $a_{1} a_{0}>0$, where

$$
\left\{\begin{array}{l}
a_{2}=\rho\left(1-2 x_{4}\right)-p^{\prime}\left(x_{4}\right) y_{4}-\lambda z_{4} \\
\quad+\lambda x_{4}-\gamma-m_{1} p^{\prime}\left(z_{4}\right) y_{4} \\
a_{1}=-\left[\rho\left(1-2 x_{4}\right)-p^{\prime}\left(x_{4}\right) y_{4}-\lambda z_{4}\right] \times \\
\quad\left[\lambda x_{4}-\gamma-m_{1} p^{\prime}\left(z_{4}\right) y_{4}\right] \\
\quad-\lambda m_{1} p\left(z_{4}\right) y_{4}-p\left(x_{4}\right) p^{\prime}\left(x_{4}\right) y_{4} \\
a_{0}=-\left[\rho\left(1-2 x_{4}\right)-p^{\prime}\left(x_{4}\right) y_{4}-\lambda z_{4}\right] \times \\
{\left[\lambda x_{4}-\gamma-m_{1} p^{\prime}\left(z_{4}\right) y_{4}\right]+\lambda m_{2} p\left(x_{4}\right) p^{\prime}\left(z_{4}\right) y_{4} z_{4}} \\
+p^{\prime}\left(x_{4}\right) y_{4} m_{1} p\left(z_{4}\right)\left(\lambda x_{4}-\gamma\right) \\
+p\left(x_{4}\right) p^{\prime}\left(x_{4}\right) y_{4}\left(\lambda x_{4}-\gamma-m_{1} p^{\prime}\left(z_{4}\right) y_{4}\right)
\end{array}\right.
$$

Proof: The eigenvalues of the jacobian matrix $J\left(B_{0}\right)$ are $\chi_{1}=0 ; \chi_{2}=\lambda x_{0}-\gamma$ and $\chi_{3}=p\left(x_{0}\right)-d$.

a) If $\frac{\gamma}{\lambda}<\frac{1}{2}$ or $d<\frac{1}{a+2 b+4}=p\left(x_{0}\right)$, then $\chi_{2}>0$ or $\chi_{3}>0$ and $B_{0}$ is unstable.

b) If $\frac{\gamma}{\lambda}>\frac{1}{2}$ and $d=\frac{1}{a+2 b+4}=p\left(x_{0}\right)$, then $\chi_{2}<0$ and $\chi_{3}=0$. Hence, the stability of $B_{0}$ is given by the center manifold theorem. The translation $\left(u_{1}, u_{2}, u_{3}\right)=\left(x-x_{0}, z, y\right)$ brings the singular point $B_{0}$ to the origin. In the neighborhood of the origin and, since $h_{1}=\frac{\rho}{4}$, System 1 has a new form. The Jacobian matrix $J\left(B_{0}\right)$ is not diagonalizable and the passage matrix to the Jordan's basis is 
J. Tewa et al., Predator-Prey Model with Prey Harvesting, Holling Response Function of Type III...

$P=\left(\begin{array}{ccc}-1 & 0 & 1 \\ 0 & 0 & -1 \\ 0 & 1 & 0\end{array}\right)$. By the transformation $\left(v_{1}, v_{2}, v_{3}\right)^{T}=P^{-1}\left(u_{1}, u_{2}, u_{3}\right)^{T}$, the system becomes:

$$
\left\{\begin{array}{l}
\dot{v}_{1}=v_{2}+p^{\prime}\left(x_{0}\right)\left(v_{1} v_{3}-v_{3}^{2}\right) \\
+\frac{p^{\prime \prime}\left(x_{0}\right)}{2}\left(v_{1}^{2}+v_{3}^{2}-2 v_{1} v_{3}\right) v_{2} \\
+\frac{m_{1} p^{\prime \prime}(0)}{2} v_{2} v_{3}^{2}+O\left(\left|\left(v_{1}, v_{2}, v_{3}\right)\right|^{4}\right), \\
\dot{v}_{2}=v_{3}+p^{\prime}\left(x_{0}\right)\left(v_{1} v_{3}-v_{3}^{2}\right) \\
+\frac{p^{\prime \prime}\left(x_{0}\right)}{2}\left(v_{1}^{2}+v_{3}^{2}-2 v_{1} v_{3}\right) v_{2} \\
-\frac{m_{2} p^{\prime \prime}(0)}{2} v_{2} v_{3}^{2}+O\left(\left|\left(v_{1}, v_{2}, v_{3}\right)\right|^{4}\right), \\
\dot{v}_{3}=\chi_{2} v_{3}-\lambda\left(v_{1} v_{3}-v_{3}^{2}\right)+\frac{m_{1} p^{\prime \prime}(0)}{2} v_{2} v_{3}^{2} \\
+O\left(\left|\left(v_{1}, v_{2}, v_{3}\right)\right|^{4}\right) .
\end{array}\right.
$$

We can now find that the center manifold is given by $W^{c}=\left\{v_{3}=0\right\}$. Therefore, the system (6) is topologically equivalent, around the origin, to the following system:

$$
\left\{\begin{array}{l}
\dot{v}_{1}=v_{2}+\frac{p^{\prime \prime}\left(x_{0}\right)}{2} v_{1}^{2} v_{2}+O\left(\left|\left(v_{1}, v_{2}\right)\right|^{4}\right), \\
\dot{v}_{2}=O\left(\left|\left(v_{1}, v_{2}\right)\right|^{4}\right), \\
\dot{v}_{3}=O\left(\left|\left(v_{1}, v_{2}\right)\right|^{4}\right) .
\end{array}\right.
$$

Then, the singular point $B_{0}$ is unstable.

c) If $\frac{\gamma}{\lambda}=\frac{1}{2}$ and $d=\frac{1}{a+2 b+4}=p\left(x_{0}\right)$, then $\chi_{2}=0$ and $\chi_{3}=0$. Applying the center manifold theory as previously, $B_{0}$ is unstable.

d) If $\frac{\gamma}{\lambda}=\frac{1}{2}$ and $d>\frac{1}{a+2 b+4}=p\left(x_{0}\right)$, we have $\chi_{2}=0$ and $\chi_{3}<0$. Applying the center manifold theory as previously, $B_{0}$ is unstable.

The stability of $B_{1}$ is obtained with jacobian matrix. The stability of $B_{2}$ is obtained using the center manifold theorem. Taking into account the fact that $p\left(x_{3}\right)=d$, one find that the characteristic polynomial of the linearized system around the singular point $B_{3}$ is

$$
\begin{aligned}
Q(\chi) & =\left(\chi-\lambda x_{3}+\gamma\right)\left[-\chi^{2}+\left(\rho\left(1-2 x_{3}\right)-p^{\prime}\left(x_{3}\right) y_{3}\right) \chi\right] \\
& -d\left(\chi-\lambda x_{3}+\gamma\right) p^{\prime}\left(x_{3}\right) y_{3} .
\end{aligned}
$$

The discriminant of $Q(\chi)$ is

$$
\Delta_{3}\left(h_{1}\right)=\left(\rho\left(1-2 x_{3}\right)-p^{\prime}\left(x_{3}\right) y_{3}\right)^{2}-4 d p^{\prime}\left(x_{3}\right) y_{3} .
$$

a) If $x_{3}>\frac{\gamma}{\lambda}$, then the eigenvalue $\chi_{1}=\lambda x_{3}-\gamma>$ 0 . Hence, $B_{3}$ is unstable.

b) If $x_{3}<\frac{\gamma}{\lambda}$, then $\chi_{1}<0$. b1) When $\Delta_{3}\left(h_{1}\right)=0$ the Jacobian matrix at $B_{3}$ has a double eigenvalue

$$
\chi_{0}\left(x_{3}\right):=\frac{\rho\left(1-2 x_{3}\right)-p^{\prime}\left(x_{3}\right) y_{3}}{2} .
$$

- If $d \geq \frac{1}{a+2 b+4}$, then $x_{3}>\frac{1}{2}$. From where $\chi_{0}\left(h_{1}\right)<0$. Therefore, the singular point $B_{3}$ is stable.

- If $d<\frac{1}{a+2 b+4}$, then: When $\chi_{0}\left(h_{1}\right)<0$ (resp. $\chi_{0}\left(h_{1}\right)>0$ ) the singular point $B_{3}$ is stable (resp. unstable).

b2) When $\Delta_{3}>0$ the eigenvalues of the Jacobian matrix at $B_{3}$ are $\chi_{1}<0, \chi_{2}=\chi_{0}\left(h_{1}\right)-\frac{\sqrt{\Delta_{3}}}{2}$ and $\chi_{3}=\chi_{0}\left(h_{1}\right)+\frac{\sqrt{\Delta_{3}}}{2}$. We have, $\chi_{2} \chi_{3}=$ $d p^{\prime}\left(h_{1}\right) y_{3}>0$ and $\chi_{2}+\chi_{3}=\chi_{0}\left(h_{1}\right)$, where $\chi_{0}\left(h_{1}\right)$ is defined by 8 .

- If $d \geq \frac{1}{a+2 b+4}$, then the singular point $B_{3}$ is stable.

- If $d<\frac{1}{a+2 b+4}$, then: When $\chi_{0}\left(h_{1}\right)<0$ (resp. $\chi_{0}\left(h_{1}\right)>0$ ) the singular point $B_{3}$ is stable (resp. unstable).

b3) If $\Delta_{3}<0$, then the eigenvalues of the Jacobian matrix at $B_{3}$ are $\chi_{1}<0, \chi_{2}=\chi_{0}\left(h_{1}\right)-$ $i \frac{\sqrt{-\Delta_{3}}}{2}$ and $\chi_{3}=\chi_{0}\left(h_{1}\right)+i \frac{\sqrt{-\Delta_{3}}}{2}$, where $\chi_{0}\left(h_{1}\right)$ is defined by $\left\{8\right.$. If $d \geq \frac{1}{a+2 b+4}$, then the singular point $B_{3}$ is stable. If $d<$ $\frac{1}{a+2 b+4}$ and $\chi_{0}\left(h_{1}\right)<0$ then, the singular point $B_{3}$ is stable. If $d<\frac{1}{a+2 b+4}$ and $\chi_{0}\left(h_{1}\right)>0$ then, the singular point is unstable. If $d<\frac{1}{a+2 b+4}$ and $\chi_{0}\left(h_{1}\right)=0$ then, the real central and stable spaces are respectively defined by $E^{c}=\langle(1,0,0) ;(0,0,1)\rangle$ and $E^{s}=\left\langle\left(1,-1-\frac{d p^{\prime}\left(x_{3}\right) y_{3}}{\chi_{1}^{2}}, \frac{p^{\prime}\left(x_{3}\right) y_{3}}{\chi_{1}}\right)\right\rangle$. Then applying the center manifold theorem it comes that the singular point $B_{3}$ is unstable.

The stability of $B_{4}$ is obtained using the Routh-Hurwitz conditions. 
B. Results for the Model (2) with Disease only inside Predator Population

Let us set $u_{2}(x)=\frac{e}{\delta}\left[\frac{\mu_{1}}{e}-p(x)\right]$ and $v_{2}(x)=$ $\frac{(p(x)-d) u_{2}(x)}{e\left[\frac{d}{e}-p(x)\right]}$. Let $x_{5}$ the eventual positive root of equation $p\left(x_{5}\right)=\frac{d}{e}$ and the function $g_{2}(x)=\rho x(1-$ $x)-h_{1}-p(x) u_{2}(x)-\eta_{1} p(x) v_{2}(x)$.

Hypothesis 1 : The attack of non-infected predators is more important than the one of the infected predators i.e. $e=\frac{\tilde{e}}{\tilde{c}} \leq 1$.

Theorem 3: The equilibria of System (2), where $x_{0}$; $x_{1}$ and $x_{2}$ are given by (4), according to the values of the parameters, are given as follow.

- When $h_{1}>\frac{\rho}{g}$, then there is no equilibrium point.

- When $h_{1}=\frac{\rho}{4}$, then $C_{0}\left(x_{0} ; 0 ; 0\right)$ is a double point if $d \neq \frac{1}{a+2 b+4}$ and triple point if $d=\frac{1}{a+2 b+4}$.

- When $h_{1}<\frac{\rho}{4}$ and $a d \geq 1$, then $C_{1}\left(x_{1} ; 0 ; 0\right)$ and $C_{2}\left(x_{2} ; 0 ; 0\right)$ exist.

- When $h_{1}<\frac{\rho}{4}$; ad $<1$ and $x_{3}=x_{1}$, then $C_{1}\left(x_{1} ; 0 ; 0\right)$ is a double point and $C_{2}\left(x_{2} ; 0 ; 0\right)$ exists.

- When $h_{1}<\frac{\rho}{4}$; ad $<1$ and $x_{3}=x_{2}$, then $C_{1}\left(x_{1} ; 0 ; 0\right)$ exists and $C_{2}\left(x_{2} ; 0 ; 0\right)$ is a double point.

- When $h_{1}<\frac{\rho}{4} ;$ ad $<1$ and $\left.x_{3} \in\right] x_{1} ; x_{2}$ [, then the equilibria are $C_{1}\left(x_{1} ; 0 ; 0\right) ; C_{2}\left(x_{2} ; 0 ; 0\right)$ and $C_{3}\left(x_{3} ; y_{3} ; 0\right)$, where $y_{3}=\frac{\rho x_{3}\left(1-x_{3}\right)-h_{1}}{d}>0$.

- When $h_{1}<\frac{\rho}{4} ; a d<1$ and $x_{3} \in\left[0 ; x_{1}[\cup] x_{2} ;+\infty[\right.$, then the equilibria are $C_{1}\left(x_{1} ; 0 ; 0\right)$ and $C_{2}\left(x_{2} ; 0 ; 0\right)$. - When $h_{1}<\frac{\rho}{4} ;$ ad $<1 ; \frac{a d}{e} \geq 1, x_{6} \in$ ]$x_{1} ; x_{2}[\cap] x_{3} ;+\infty\left[; x_{2}>x_{3}\right.$ or $h_{1}<\frac{\rho}{4} ; \frac{a d}{e}<$ $\left.1, x_{6} \in\right] x_{1} ; x_{2}[\cap] x_{3} ; x_{5}\left[; x_{2}>x_{3} ; x_{1}<x_{5}\right.$, then the equilibria are $C_{1}\left(x_{1} ; 0 ; 0\right) ; C_{2}\left(x_{2} ; 0 ; 0\right)$ and $C_{4}\left(x_{6} ; y_{6} ; \omega_{6}\right), y_{6}=u_{2}\left(x_{6}\right)$ and $\omega_{6}=v_{2}\left(x_{6}\right)$.

Proof : The equilibria $C_{0}, C_{1}, C_{2}$ and $C_{3}$ are obtained in the same way as in theorem 1 , setting the right hand side of the system equals to zero. Equilibrium $C_{4}$ exists when the previous conditions are satisfied.

Concerning the Stability analysis of these equilibria, the following theorem holds.

Theorem 4: Let's consider the System (2) and suppose that Hypothesis 1 holds.
- The equilibria $C_{0}$ and $C_{1}$ are always unstable.

- The equilibrium $C_{2}$ is stable if $h_{1}<\frac{\rho}{4}$ and $p\left(x_{2}\right)<$ $d$.

- The equilibrium $C_{3}$ is stable if and only if one of these conditions is satisfied : $h_{1}<\frac{\rho}{4}$, ad $<1, x_{3} \in$ ]$x_{1} ; x_{2}\left[\right.$ and $y_{3}=\frac{e}{\delta_{1}}\left(\frac{\mu_{1}}{e}-d\right)$ or $h_{1}<\frac{\rho}{4}, a d<1$, $\left.x_{3} \in\right] x_{1} ; x_{2}\left[, y_{3}<\frac{e}{\delta_{1}}\left(\frac{\mu_{1}}{e}-d\right), d>p\left(x_{0}\right)\right.$, or $\left.h_{1}<\frac{\rho}{4}, a d<1, x_{3} \in\right] x_{1} ; x_{2}\left[, y_{3}<\frac{e}{\delta_{1}}\left(\frac{\mu_{1}}{e}-d\right)\right.$, $d<p\left(x_{0}\right), \xi_{0}\left(x_{3}\right)<0$.

- The singular point $C_{4}\left(x_{6}, y_{6}, \omega_{6}\right)$ is asymptotically stable if and only if the following conditions are satisfied : $b_{2}<0 ; b_{2} b_{1}+b_{0}>0$ and $b_{1} b_{0}>0$, where

$$
\left\{\begin{array}{l}
b_{2}=\rho\left(1-2 x_{6}\right)-p^{\prime}\left(x_{6}\right)\left(y_{6}+\eta_{1} \omega_{6}\right) \\
\quad+p\left(x_{6}\right)-d-\delta \omega_{6} \\
b_{1}=-\left(\rho\left(1-2 x_{6}\right)-p^{\prime}\left(x_{6}\right)\left(y_{6}+\eta_{1} \omega_{6}\right)\right) \times \\
\quad\left(p\left(x_{6}\right)-d-\delta \omega_{6}\right)+\delta_{1} \omega_{6}\left(\mu-\delta y_{6}\right) \\
\quad-p\left(x_{6}\right) p^{\prime}\left(x_{6}\right) y_{6}-e \eta_{1} p\left(x_{6}\right) p^{\prime}\left(x_{6}\right) \omega_{6} \\
\\
b_{0}=e p\left(x_{6}\right) p^{\prime}\left(x_{6}\right) \omega_{6}\left[\delta y_{6}-\mu+\eta_{1}\left(p\left(x_{6}\right)-d-\delta \omega_{6}\right)\right] \\
-\delta_{1} \eta_{1} p\left(x_{6}\right) p^{\prime}\left(x_{6}\right) y_{6} \omega_{6} \\
-\delta_{1} \omega_{6}\left(\mu-\delta y_{6}\right)\left(\rho\left(1-2 x_{6}\right)-p^{\prime}\left(x_{6}\right)\left(y_{6}+\eta_{1} \omega_{6}\right)\right) .
\end{array}\right.
$$

Proof: The stability of $C_{0}$ is deduce as for $B_{0}$ in theorem 2. The jacobian matrix always has a positive eigenvalue. Then, $C_{1}$ is unstable. We obtain the stability of $C_{2}$ and $C_{3}$ applying the same arguments as for $B_{2}$ and $B_{3}$ in theorem 2. The stability of $C_{4}$ is obtained using the Routh-Hurwitz conditions.

\section{HOPF BIFURCATION}

Let us introduce the following parameters

$$
h_{10}=\frac{\rho x_{3}}{b x_{3}+2}\left[2 a x_{3}^{3}+(b-a) x_{3}^{2}+1\right] \text {, }
$$

and

$$
\Pi=\frac{1}{16}\left[p^{(2)}\left(x_{3}\right)+p^{(3)}\left(x_{3}\right)\right]-\frac{\left(p^{\prime}\left(x_{3}\right)\right)^{2}}{4 \sqrt{-\Delta_{3}\left(h_{10}\right)}} .
$$

Recalling (4), the flow of System (1) and System (2) respectively undergo a supercritical Hopf bifurcation around $h_{10}$ given by the following result

Theorem 5: (Hopf bifurcation) Let $h_{1}<\frac{\rho}{4}$; ad $<1$; $\left.x_{3} \in\right] x_{1}, \min \left(\frac{1}{2}, \frac{\gamma}{\lambda}\right)[$. Thanks to Hypothesis 1 . Then, a unique stable curve of periodic solution bifurcates from the singular points $B_{3}$ and $C_{3}$ into the regions $h_{1}>h_{10}$ if $\Pi<0$ or $h_{1}<h_{10}$ if $\Pi>0$. The singular points $B_{3}$ and $C_{3}$ are stable for $h_{1}<h_{10}$ and unstable for 
$h_{1} \geq h_{10}$. This correspond to supercritical stable Hopf bifurcation.

Proof : The proof can be obtained as in [13].

\section{Numerical Simulations}
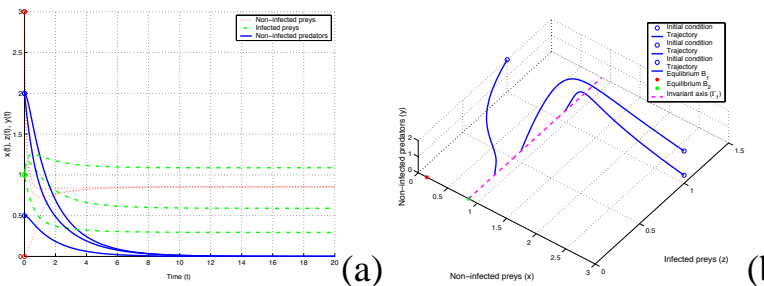

(b)

Fig. 1. Phase portraits of System $\left\{1\right.$ for $h_{1}<\frac{\rho}{4} ; \frac{\gamma}{\lambda}=x_{2}$ and $p\left(x_{2}\right)<d . B_{1}$ and $B_{2}$ are unstable. The axis $x=\frac{4}{\lambda}$ is stable.

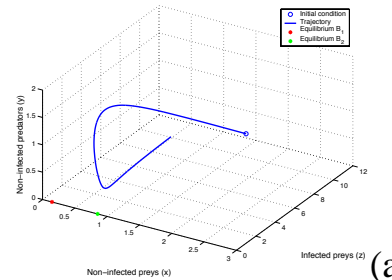

(a)

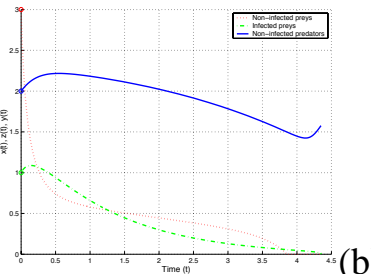

${ }^{4}(\mathrm{~b})$

Fig. 2. Phase portraits of System (1). The case (a) corresponds to $h_{1}<\frac{\rho}{4} ; \frac{\gamma}{\lambda}<x_{2}$ and $p\left(x_{2}\right)<d$. The case (b) corresponds to $h_{1}=\frac{\rho}{4} ; \frac{\gamma}{\lambda}>1 / 2$ and $d=\frac{1}{a+2 b+4}$. Unstability of $B_{1}$ and $B_{2}$.
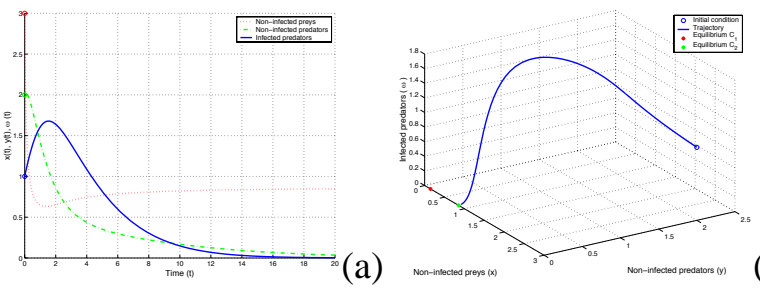

(b)

Fig. 3. Phase portraits of System 2 for $h_{1}<\frac{\rho}{4}$ and $d>p\left(x_{2}\right)$. Stability of $C_{2}$.
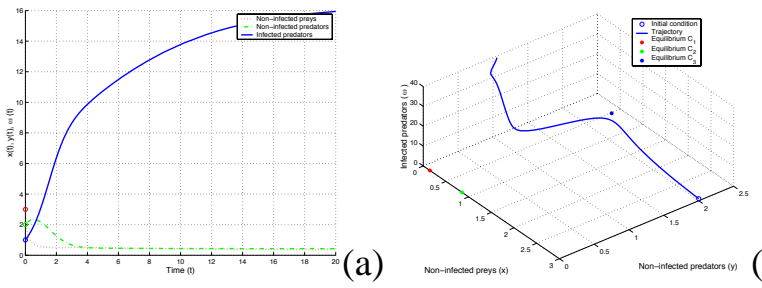

(b)

Fig. 4. Phase portraits of System $\sqrt{2}$ for $h_{1}<\frac{\rho}{4}$ and $d<p\left(x_{2}\right)$. Unstability of $C_{1}, C_{2}$ and $C_{3}$.

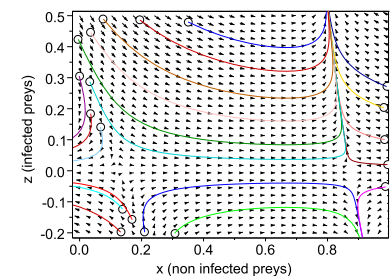

(a)

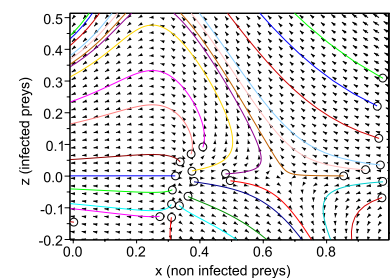

(b)

Fig. 5. Phase portraits of System (1). The case (a) corresponds to $h_{1}<\rho \frac{\gamma}{\lambda}\left(1-\frac{\gamma}{\lambda}\right)$. The case (b) corresponds to $h_{1}>\rho \frac{\gamma}{\lambda}\left(1-\frac{\gamma}{\lambda}\right)$ and $\frac{\gamma}{\lambda}<\frac{1}{2}$. Illustration of saddle-node bifurcation phenomenon.

\section{CONCLUSION}

Our goal was to analyze the modifications on a predator prey model (generalized Gause model) with prey harvesting and Holling response type III : $\frac{m x^{2}}{a x^{2}+b x+1}$, to account for a disease spreading among one of the two species. The simple epidemiological model SIS has been chosen, where only susceptibles and infectives are counted. The results indicate that either the disease dies out, leaving only neutral cycles of generalized Gause model, or one species disappears and all individuals in the other one eventually become infected. For some particular choices of the parameters however, endemic equilibria in which both populations survive seem to arise.

\section{REFERENCES}

[1] R.M. Etoua and C. Rousseau, Bifurcation analysis of a generalized Gause model with prey harvesting and a generalized Holling response function of type III , J. Differ. Equations 249, No. 9, 2316-2356 (2010), ISSN 0022-0396.

[2] A.D. Bazykin, A. Iosifovich Khibnik and B. Krauskopf, Nonlinear Dynamics of Interacting Populations, World Scientific, 1998, 193 pages.

[3] K.P. Hadeler and H.I. Freedman, Predator-prey populations with parasitic infection, J. Math. Biol. 27, (1989) 609-631. http://dx.doi.org/10.1007/BF00276947

[4] E. Venturino, The influence of diseases on Lotka-Volterra systems, Rocky Mt. J. Math. 24, (1994) 381402.

[5] R. Anguelov, Y. Dumont, J. M. S. Lubuma and M. Shillor, Comparison of some standard and nonstandard numerical methods for the MSEIR epidemiological model, Proceedings of the International Conference of Numerical Analysis and Applied Mathematics, Crete, Greece, 18-22 September 2009, American 
J. Tewa et al., Predator-Prey Model with Prey Harvesting, Holling Response Function of Type III...

Institute of Physics Conference Proceedings-AIP 1168, Volume 2, (2009) 1209-1212.

[6] J.J. Tewa, R. Fokouop, B. Mewoli and S. Bowong, Mathematical analysis of a general class of ordinary differential equations coming from within-hosts models of malaria with immune effectors, Applied Mathematics and Computation 218, (2012) 7347-7361. http://dx.doi.org/10.1016/j.amc.2011.10.085

[7] J. J. Tewa, S. Bowong, C. S. Oukouomi Noutchie; Mathematical analysis of two-patch model of tuberculosis disease with staged progression, Applied Mathematical Modelling 36, (2012) 5792 5807.

[8] M. Haque, D. Greenhalgh, When predator avoids infected prey: A model based theoretical studies. IMA J. Math. Med. Biol. 27 (2009) 75-94. http://dx.doi.org/10.1093/imammb/dqp007

[9] N. Bairagi, P. K. Roy, J. Chattopadhyay; Role of infection on the stability of a predator-prey system with several response
functions-A comparative study, Journal of Theoretical Biology, 248 (1), (2007) 10-25, ISSN 0022-5193.

[10] Krishna Padas Das, Kusumika Kundu, J. Chattopadhyay, A predator-prey mathematical model with both populations affected by diseases, Ecological Complexity 8, (2011) 68-80. http://dx.doi.org/10.1016/j.ecocom.2010.04.001

[11] M. Haque, J. Zhen, E. Venturino; Rich dynamics of LotkaVolterra type predator-prey model system with viral disease in prey species; mathematical methods in the Applied Science 32, (2009) 875-898.

[12] J. J. Tewa, V. Yatat Djeumen, S. Bowong, Predator-prey model with Holling response function of type II and SIS infectious disease, Applied Mathematical Modelling, (2012) to appear.

[13] Y. A. Kuznetsov, Elements of Applied Bifurcation Theory: Third edition, Appl. Math. Sci. 112, Springer Vergal, New York, 2004. 